Wright State University

CORE Scholar

2-1-2009

\title{
Ga-Related Photoluminescence Lines in Ga-Doped ZnO Grown by Plasma-Assisted Molecular-Beam Epitaxy
}

Z. Yang

David C. Look

Wright State University - Main Campus, david.look@wright.edu

J. L. Liu

Follow this and additional works at: https://corescholar.libraries.wright.edu/physics

Part of the Physics Commons

\section{Repository Citation}

Yang, Z., Look, D. C., \& Liu, J. L. (2009). Ga-Related Photoluminescence Lines in Ga-Doped ZnO Grown by Plasma-Assisted Molecular-Beam Epitaxy. Applied Physics Letters, 94 (7), 72101.

https://corescholar.libraries.wright.edu/physics/99

This Article is brought to you for free and open access by the Physics at CORE Scholar. It has been accepted for inclusion in Physics Faculty Publications by an authorized administrator of CORE Scholar. For more information, please contact library-corescholar@wright.edu. 


\title{
Ga-related photoluminescence lines in Ga-doped ZnO grown by plasma-assisted molecular-beam epitaxy
}

\author{
Z. Yang, ${ }^{1}$ D. C. Look, ${ }^{2}$ and J. L. Liu ${ }^{1, a)}$ \\ ${ }^{1}$ Department of Electrical Engineering, Quantum Structures Laboratory, University of California \\ at Riverside, Riverside, California 92521, USA \\ ${ }^{2}$ Semiconductor Research Center, Wright State University, Dayton, Ohio 45435, USA
}

(Received 25 November 2008; accepted 19 January 2009; published online 17 February 2009)

Low-temperature photoluminescence (PL) and temperature-dependent Hall-effect (T-Hall) measurements were carried out in undoped and $\mathrm{Ga}$-doped $\mathrm{ZnO}$ thin films grown by molecular-beam epitaxy. As the carrier concentration increases from $1.8 \times 10^{18}$ to $1.8 \times 10^{20} \mathrm{~cm}^{-3}$, the dominant PL line at $9 \mathrm{~K}$ changes from $I_{1}(3.368-3.371 \mathrm{eV})$ to $I_{D A}(3.317-3.321 \mathrm{eV})$, and finally to $I_{8}(3.359 \mathrm{eV})$. The dominance of $I_{1}$, due to ionized-donor bound excitons, is unexpected in $n$-type samples but is shown to be consistent with the T-Hall results. We also show that $I_{D A}$ has characteristics of a donor-acceptor-pair transition, and use a detailed, quantitative analysis to argue that it arises from $\mathrm{Ga}_{\mathrm{Zn}}$ donors paired with Zn-vacancy $\left(V_{\mathrm{Zn}}\right)$ acceptors. In this analysis, the $\mathrm{Ga}_{\mathrm{Zn}}^{0 /+}$ energy is well-known from two-electron satellite transitions, and the $V_{\mathrm{Zn}}^{0 /-}$ energy is taken from a recent theoretical calculation. (C) 2009 American Institute of Physics. [DOI: 10.1063/1.3080204]

$\mathrm{ZnO}$ materials have potential applications in optoelectronics and spintronics. ${ }^{1-4}$ Although $p$-type doping has attracted more recent attention in $\mathrm{ZnO}$ research, $n$-type materials with high crystallinity and controllable electron carrier concentration $(n)$ are also indispensable toward the applications. For example, controllable $n$ is important for $\mathrm{ZnO}$ based dilute magnetic semiconductor materials because their magnetic properties can be modulated by $n .^{5-7}$ Also, groupIII-doped $\mathrm{ZnO}$ with large $n$ is a potential candidate for replacing conventional transparent conducting oxides such as indium tin oxide. Among group-III elements, Ga is an excellent $n$-type dopant in $\mathrm{ZnO}$ with a more compatible covalent bond length (1.92 $\AA$ for $\mathrm{Ga}-\mathrm{O}$ and $1.97 \AA$ for $\mathrm{Zn}-\mathrm{O})$ than that of $\mathrm{Al}$ or In $(2.7 \AA$ for $\mathrm{Al}-\mathrm{O}$ and $2.1 \AA$ for $\mathrm{In}-\mathrm{O})$.

Ga-doped $\mathrm{ZnO}$ has been widely studied $;^{8-14}$ however, among the Ga-related excitonic transitions, only excitons bound to neutral $\mathrm{Ga}_{\mathrm{Zn}}$ donors ( $I_{8}$ in the literature) have been commonly reported. This is in part due to the strong tendency for ionized donors to become neutral under light excitation because of the reaction $D^{+}+e^{-} \rightarrow D^{0}$. In this paper, we report systematic photoluminescence (PL) studies of high-quality Ga-doped $\mathrm{ZnO}$ thin films with various $n$. Variations in the energies and strengths of three dominant donorrelated PL emissions with increasing $n$ are discussed in detail.

$\mathrm{ZnO}$ thin films were grown on $r$-plane sapphire substrates using plasma-assisted molecular-beam epitaxy (MBE). An undoped sample (A) and eight Ga-doped samples (B-I), with room-temperature electron carrier concentration $n$ ranging from $1.9 \times 10^{18}$ to $1.8 \times 10^{20} \mathrm{~cm}^{-3}$, were prepared. The $n$ of each sample was tuned by the Ga incorporation controlled by the Ga effusion cell temperature. The $300 \mathrm{~K}$ $(10 \mathrm{~K}) n$ values of samples A-I are $0.19(0.18), 0.55(0.36)$, 0.96 (0.88), 1.5 (1.5), 2.3 (2.2), 2.5 (2.4), 3.6 (3.5), 7.4 (7.4), and 18 (18), respectively, in unit $10^{19} \mathrm{~cm}^{-3}$. The $n$ values do

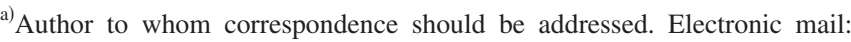
jianlin@ee.ucr.edu.
}

not show strong temperature dependence since they are basically degenerate.

Reflection high-energy electron diffraction (RHEED) measurements were performed in situ on the as-grown samples in the MBE system. Hall-effect measurements were carried out using a Quantum Design physical properties measurement system at 10 and $300 \mathrm{~K}$, and a LakeShore 7507 system from 15 to $320 \mathrm{~K}$. X-ray diffraction (XRD) measurements were performed using a Bruker D8 Advance X-ray diffractometer. PL measurements were carried out using a home-built PL system with temperature control over a range of $8.5-300 \mathrm{~K}$. The $325 \mathrm{~nm}$ wavelength of a He-Cd laser was used as an excitation source and a photomultiplier tube was used to detect the PL signals. The resolution of the PL system was $0.15 \mathrm{~nm}$, which is about $1.5 \mathrm{meV}$ in the ultraviolet emission region.

Figure 1 shows the XRD spectra of undoped sample A and heavily Ga-doped sample $\mathrm{G}$. Only the $\mathrm{ZnO}$ and $\mathrm{GaZnO}$ $(11 \overline{2} 0)$ peaks are observed in the samples, indicating that both samples are single crystalline and the heavy Ga doping does not significantly degrade the crystallinity of the film. The insets in Fig. 1 show the RHEED patterns of the two samples. Note the change from the streaky pattern of the undoped sample to the spotty pattern of the Ga-doped

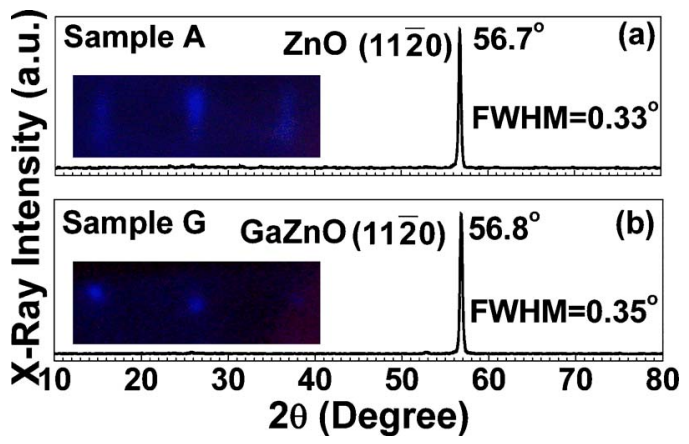

FIG. 1. (Color online) XRD patterns of (a) undoped $\mathrm{ZnO}$ sample A and (b) heavy-Ga-doped $\mathrm{ZnO}$ sample $\mathrm{G}$. The insets show the RHEED patterns of samples A and G, which are streaky and spotty, respectively. 


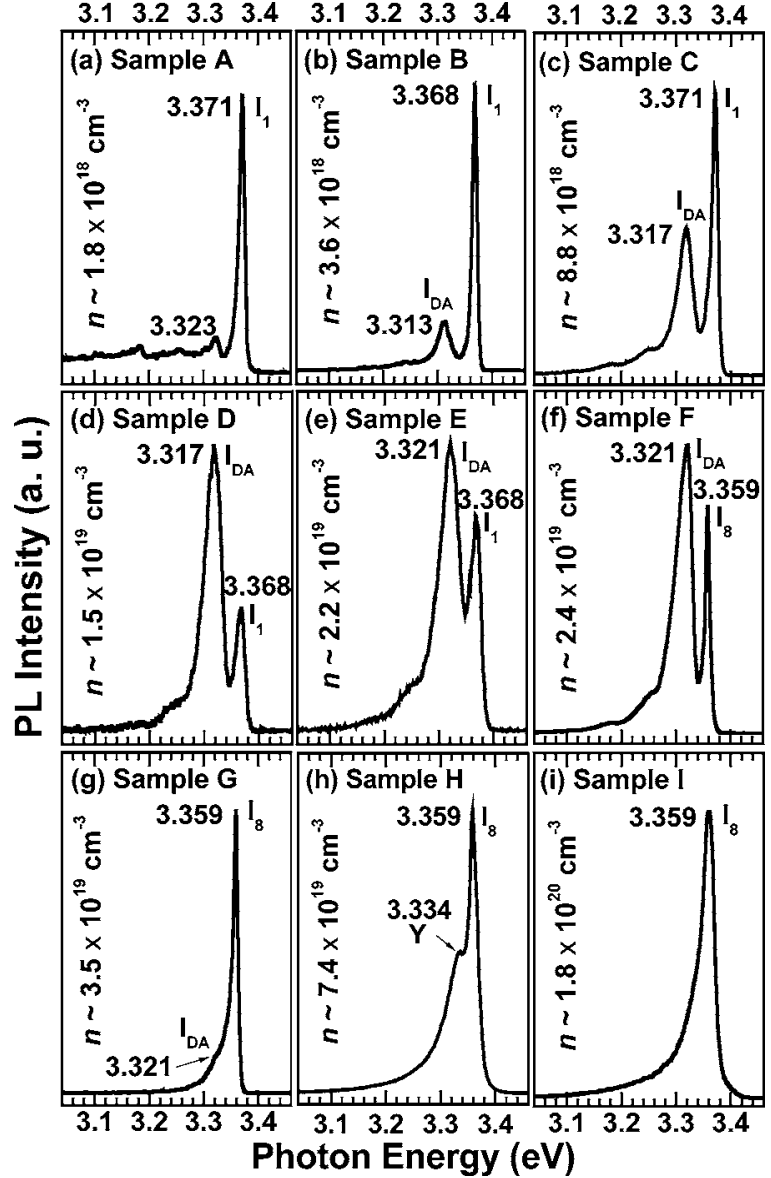

FIG. 2. PL spectra measured at $9 \mathrm{~K}$ for samples A-I [(a)-(i)].

sample, which indicates that the surface of the film becomes rougher after heavy Ga doping.

Figures 2(a)-2(i) show the 9 K PL spectra of samples A to I. In the nine spectra, three basic PL peaks, designated $I_{1}$, $I_{8}$, and $I_{D A}$, are dominant: $I_{1}$, at $3.368-3.371 \mathrm{eV}$, is found in samples A to E; $I_{8}$, at $3.359 \mathrm{eV}$, in samples $\mathrm{F}$ to $\mathrm{I}$; and $I_{D A}$, at $3.313-3.321 \mathrm{eV}$, in samples B to G. The $I_{8}$ line is the commonly accepted as the neutral $\mathrm{Ga}$ donor-bound-exciton recombination. ${ }^{8,15,16}$ The $I_{1}$ line lies above the common neutral donor-bound-exciton lines, $I_{9}(\mathrm{In}), I_{8}(\mathrm{Ga}), I_{6}(\mathrm{Al})$, and $I_{4}(\mathrm{H})$, which span the range of 3.357-3.363 eV but below the free A exciton line at $3.377 \mathrm{eV}$. It has been seen in the past $^{16,17}$ but never unambiguously identified. More recently, however, it has been associated with an exciton bound to an ionized Ga donor. ${ }^{18,19}$ In almost all $n$-type $\mathrm{ZnO}$ samples, the intensity of $I_{1}$ is much less than that of $I_{8}$; however, several of our samples show exactly the opposite relationship, and we will give the reasons below.

The PL lines occurring in the region of $3.30-3.32 \mathrm{eV}$ have been variously assigned in the literature to many different transitions, including those involving acceptor-bound excitons, donor-acceptor pairs (DAPs), and free electrons to neutral acceptors. ${ }^{20}$ In fact, more than one mechanism may contribute to luminescence in this region. Here we will argue that DAP transitions best describe line $I_{D A}$ in our case. Immediate support for this assignment arises from a study of the excitation-power dependence of the luminescence in sample E, Fig. 2. In this sample, the $I_{D A}$ transition energy clearly blueshifts with excitation power (not shown), whereas that of $I_{1}$ does not. This is a strong indication that
$I_{D A}$ represents a DAP transition. Further support is presented below.

We propose that $I_{D A}$ consists of transitions between neutral $\mathrm{Ga}_{\mathrm{Zn}}$ donors and neutral $\mathrm{Zn}$-vacancy $\left(V_{\mathrm{Zn}}\right)$ acceptors, or acceptor complexes. Obviously $\mathrm{Ga}_{\mathrm{Zn}}$ donors should be abundant in our samples but $V_{\mathrm{Zn}}$ acceptors also have low formation energies and are common in $n$-type $\mathrm{ZnO} .{ }^{21}$ In this scenario, the DAP transition would be written as $\mathrm{Ga}_{\mathrm{Zn}}^{0}+V_{\mathrm{Zn}}^{0}$ $\rightarrow \mathrm{Ga}_{\mathrm{Zn}}^{+}+V_{\mathrm{Zn}}$. The photon emitted in this transition should have an energy

$$
E_{D A}=E_{g}-E_{D}\left(G a_{\mathrm{Zn}}^{0 /+}\right)-E_{A}\left(V_{\mathrm{Zn}}^{0 /-}\right)+E_{\mathrm{coul}}-E_{\mathrm{vdW}},
$$

where $E_{D}\left(\mathrm{Ga}_{\mathrm{Zn}}\right)=55 \mathrm{meV}$ from analysis of two-electron satellite spectra ${ }^{15,16}$ and $E_{A}\left(V_{\mathrm{Zn}}\right)$ is estimated to be about $180 \mathrm{meV}$, as recently determined from density-functional theory (DFT). ${ }^{22}$ The term $E_{\text {coul }}=e^{2} / 4 \pi \varepsilon r_{D A}$ is the Coulomb energy arising from the proximity between the donor and acceptor, and $E_{\mathrm{vdW}}$ is the van der Waals (vdW) polarization energy associated with the dipole-dipole interaction. ${ }^{23}$ The $\mathrm{vdW}$ term is very small compared to the Coulomb term when $r_{D A}$ is large, and hence is generally neglected. However, when $r_{D A}$ is small (say, $<2 \mathrm{~nm}$ ), the contribution from the $\mathrm{vdW}$ term needs to be considered. The traditional vdW term is in the form of

$$
E_{\mathrm{vdW}}=\frac{e^{2}}{4 \pi \varepsilon r_{D A}}\left(\frac{b}{r_{D A}}\right)^{5}
$$

as proposed by $\mathrm{Dean}^{23}$ in GaP materials, with $b$ a constant for a given donor and acceptor. However, it was later found that this equation gives a very unsatisfactory result in II-VI materials, especially in the region of closer pairs $\left(r_{D A}\right.$ $<3 \mathrm{~nm}){ }^{24}$ Instead, an exponential form

$$
E_{\mathrm{vdW}}(\mathrm{eV})=0.06823 \times \exp \left[-1.312 \times r_{D A}(\mathrm{~nm})\right]
$$

was employed by Neumark ${ }^{25}$ to achieve a good fit. We will use Neumark's formula as a reasonable approximation for our samples because $E_{\mathrm{vdW}}$ is rather small compared to $E_{\text {coul }}$ and thus high accuracy in $E_{\mathrm{vdW}}$ is not required.

Consider the PL spectrum for sample F in Fig. 2. Here $I_{8}$ is strong and $I_{1}$ does not appear in the linear plot, which indeed is typical of most $\mathrm{ZnO}$ samples that we have examined. To apply Eq. (1), we need to know the donor and acceptor concentrations, $N_{D}$ and $N_{A}$, respectively. Measurement of these quantities requires temperature-dependent Halleffect (T-Hall) measurements, and to analyze the T-Hall data, we employ a general two-layer algorithm outlined in Ref. 26. The fitting results are $N_{D 1}=6.3 \times 10^{19} \mathrm{~cm}^{-3}, N_{D 2}$ $=7.0 \times 10^{18} \mathrm{~cm}^{-3}, \quad N_{A 1}=4.0 \times 10^{19} \mathrm{~cm}^{-3}, \quad$ and $N_{A 2}=2.0$ $\times 10^{18} \mathrm{~cm}^{-3}$; and $d_{1}=440 \mathrm{~nm}$ and $d_{2}=20 \mathrm{~nm}$. Here $N_{D i}$, $N_{A i}$, and $d_{i}$, are the donor concentration, acceptor concentration, and thickness of layer $i$, respectively. Layer 2 is probably representative of the surface and/or interface regions and is not of importance in this study. In the dark, at $10 \mathrm{~K}$, the neutral donor concentration in layer 1 is $N_{D}^{0} \approx N_{D}-N_{A}$ $=2.3 \times 10^{19} \mathrm{~cm}^{-3}$, and the neutral acceptor concentration is $N_{A}^{0} \approx 0$ since almost all of the acceptors will be negatively charged. In the light, photogenerated electrons will create more $D^{0}$ through the reaction $e+D^{+} \rightarrow D^{0}$, and photogenerated holes will create $A^{0}$ through the reaction $h+A^{-} \rightarrow A^{0}$. Of course, some of the photogenerated electrons and holes will also form free excitons, $h+e \rightarrow X$, and at $10 \mathrm{~K}$ most of these excitons will bind to neutral donors, forming $D^{0} X$. (Note that 
neutral acceptors are rarely seen in $\mathrm{ZnO}$.) If these three reactions are the only ones available (or at least are dominant), then the photogenerated neutral donors and acceptors must be equal: $\Delta D^{0}=\Delta A^{0}$. Since clearly $D^{0}+\Delta D^{0}>\Delta A^{0}$, the average distance between neutral donors and acceptors will be determined by the average distance between neutral donors, i.e., $r_{D A} \approx\left(3 / 4 \pi N_{D}^{0}\right)^{1 / 3} \approx 2.2 \mathrm{~nm}$, assuming $N_{D}^{0} \gg \Delta N_{D}^{0}$. Then, applying Eqs. (1) and (3), $E_{D A}=3.437-0.055-0.18$ $+0.081-0.004=3.279 \mathrm{eV}$. If on the other hand, it is assumed that the light neutralizes all of the donors, then $E_{D A}$ $=3.307 \mathrm{eV}$. With this and other uncertainties, including that in the DFT value $\left[E_{A}\left(V_{\mathrm{Zn}}^{0 /-}\right)=0.18 \mathrm{eV}\right]$, our range of 3.279$3.307 \mathrm{eV}$ is in good agreement with the experimental value, $3.321 \mathrm{eV}$. This agreement, along with the aforementioned observation of a blue shift with excitation intensity, justifies the assignment of $I_{D A}$ as a DAP transition involving $\mathrm{Ga}_{\mathrm{Zn}}$ donors and $V_{\mathrm{Zn}}$ acceptors.

The other main PL line in Fig. 2(f), $I_{8}$, is almost universally acknowledged to be the $\mathrm{Ga}_{\mathrm{Zn}} D^{0} X$ line. The existence of this line is entirely expected from the high concentration of neutral donors available for the reaction $D^{0}+X \rightarrow D^{0} X$. Although the relative strengths of $I_{8}$ and $I_{D A}$ depend upon many factors, certainly one of these factors is the photogenerated neutral-acceptor concentration $\Delta A^{0}$. Indeed, as more Ga donors are added to the $\mathrm{ZnO}$ (cf. samples $\mathrm{G}, \mathrm{H}$, and I in Fig. 2), the reaction $e+h+D^{0} \rightarrow D^{0} X$ may become more and more dominant over the reaction $h+A^{-} \rightarrow A^{0}$, thus favoring $I_{8}$ over $I_{D A}$.

Finally, we must explain the dominance of ionized donor-bound excitons in low-Ga-doped samples, such as sample A, which has only background Ga doping. The occurrence of strong $D^{+} X$ transitions, represented by $I_{1}$, is very unusual. To explain this phenomenon, we again turn to the T-Hall fitting, ${ }^{26}$ which for sample A [Fig. 2(a)] gives $N_{D 1}=1.33 \times 10^{20} \mathrm{~cm}^{-3}, \quad N_{D 2}=6.5 \times 10^{19} \mathrm{~cm}^{-3}, \quad N_{A 1}=1.30$ $\times 10^{20} \mathrm{~cm}^{-3}$, and $N_{A 2}=2.5 \times 10^{19} \mathrm{~cm}^{-3}$ and $d_{1}=199 \mathrm{~nm}$ and $d_{2}=1 \mathrm{~nm}$. Again, only layer 1 is of any importance, and in the dark we get $N_{D}^{0}=N_{D}-N_{A} \approx 3 \times 10^{18} \mathrm{~cm}^{-3}$ and $N_{D}^{+}$ $\approx N_{A}=1.3 \times 10^{20} \mathrm{~cm}^{-3}$. (Note that the compensation ratio $N_{A} / N_{D}$ is close to one for sample A. However, this is often the case in as-grown $\mathrm{ZnO}$; see, e.g., Table I in Ref. 26.) Thus, $N_{D}^{+} \gg N_{D}^{0}$, so that in weak light the ionized donor-bound excitons $D^{+} X\left(I_{1}\right)$ might be expected to dominate over the neutral donor-bound excitons $D^{0} X\left(I_{8}\right)$. Even in stronger light, it takes three reactions to make $D^{0} X$ from $D^{+}:$(1) $e+h \rightarrow X$, (2) $e+D^{+} \rightarrow D^{0}$, and (3) $X+D^{0} \rightarrow D^{0} X$. The DAP analysis [Eq. (1)] for sample A follows that given earlier for sample $F$, and the results are that $E_{D A} \approx 3.243 \mathrm{eV}$ if there is almost no additional donor neutralization from the photoexcitation, and $E_{D A} \approx 3.334 \mathrm{eV}$ if all of the donors are neutralized and participate in DAP recombinations. The experimental value $E_{D A} \approx 3.323 \mathrm{eV}$ falls in this range; however, the accuracy of this value may be poor because the $I_{D A}$ intensity is quite weak and the line may overlap with other PL lines in this region. For example, there is a well-known line at $3.333 \mathrm{eV}$ that often appears in $\mathrm{ZnO}$. Further analysis of the relative line intensities and energies would require more detailed knowledge of the various capture cross sections and other factors and is beyond the scope of this work.

In summary, we have carried out PL and Hall-effect measurements for a series of $\mathrm{Ga}$-doped $\mathrm{ZnO}$ thin films grown by MBE. For high Ga doping, the PL spectra are dominated by the neutral-Ga donor-bound exciton $I_{8}$ at $3.359 \mathrm{eV}$, and for low Ga doping, the ionized-Ga donor bound exciton $I_{1}$ at $3.371 \mathrm{eV}$. The low-Ga spectra in this study demonstrate one of the first ever observations of higher $I_{1}$ intensity than that of $I_{8}$, and this phenomenon is explained by the T-Hall result that $\left[\mathrm{Ga}^{+}\right] \gg\left[\mathrm{Ga}^{0}\right]$ in these low-Ga samples. For intermediate Ga doping, a line that ranges from 3.31 to $3.32 \mathrm{eV}$, designated as $I_{\mathrm{DA}}$, is dominant. We have shown that $I_{\mathrm{DA}}$ has characteristics of a DAP transition, and have used a detailed, quantitative analysis to argue that it arises from $\mathrm{Ga}_{\mathrm{Zn}}$ donors paired with $\mathrm{Zn}$-vacancy $\left(V_{\mathrm{Zn}}\right)$ acceptors. The success of our analysis depends upon, and lends credence to, a recent theoretical calculation of the $V_{\mathrm{Zn}}^{0 /-}$ acceptor transition energy.

This work was supported by ONR/DMEA through the Center of Nanomaterials and Nanodevice (CNN) under the Award No. H94003-08-2-0803. D.C.L. was supported by AFOSR Grant No. FA9550-07-1-0013, NSF Grant No. DMR0513968, DOE Grant No. DE-FG02-07ER46389, and ARO Grant No. W911NF-07-D-0001/Task07275.

${ }^{1}$ D. C. Look, Mater. Sci. Eng., B 80, 383 (2001).

${ }^{2}$ S. J. Pearton, D. P. Norton, K. Ip, Y. W. Heo, and T. Steiner, Superlattices Microstruct. 34, 3 (2003).

${ }^{3}$ Ü. Özgür, Ya. I. Alivov, C. Liu, A. Teke, M. A. Reshchikov, S. Doğan, V. Avrutin, S.-J. Cho, and H. Morkoç, J. Appl. Phys. 98, 041301 (2005).

${ }^{4}$ C. Klingshirn, Phys. Status Solidi B 244, 3027 (2007).

${ }^{5}$ K. Sato and H. Katayama-Yoshida, Semicond. Sci. Technol. 17, 367 (2002).

${ }^{6}$ Z. Yang, J. L. Liu, M. Biasini, and W. P. Beyermann, Appl. Phys. Lett. 92, 042111 (2008).

${ }^{7}$ Z. Yang, M. Biasini, W. P. Beyermann, M. B. Katz, O. K. Ezekoye, X. Q. Pan, Y. Pu, J. Shi, Z. Zuo, and J. L. Liu, J. Appl. Phys. 104, 113712 (2008).

${ }^{8}$ H. J. Ko, Y. F. Chen, S. K. Hong, H. Wenisch, T. Yao, and D. C. Look, Appl. Phys. Lett. 77, 3761 (2000).

${ }^{9}$ T. Makino, Y. Segawa, S. Yoshida, A. Tsukazaki, A. Ohtomo, and M. Kawasaki, Appl. Phys. Lett. 85, 759 (2004).

${ }^{10}$ J. D. Ye, S. L. Gu, S. M. Zhu, S. M. Liu, Y. D. Zheng, R. Zhang, and Y. Shi, Appl. Phys. Lett. 86, 192111 (2005).

${ }^{11}$ F. Reuss, S. Frank, C. Kirchner, R. Kling, Th. Gruber, and A. Waag, Appl. Phys. Lett. 87, 112104 (2005).

${ }^{12}$ T. Makino, Y. Segawa, S. Yoshida, A. Tsukazaki, A. Ohtomo, and M. Kawasaki, J. Appl. Phys. 98, 093520 (2005).

${ }^{13}$ V. Bhosle, A. Tiwari, and J. Narayan, Appl. Phys. Lett. 88, 032106 (2006).

${ }^{14}$ H. C. Park, D. Byun, B. Angadi, D. H. Park, W. K. Choi, J. W. Choi, and Y. S. Jung, J. Appl. Phys. 102, 073114 (2007).

${ }^{15}$ D. C. Look, G. C. Farlow, P. Reunchan, S. Limpijumnong, S. B. Zhang, and K. Nordlund, Phys. Rev. Lett. 95, 225502 (2005).

${ }^{16}$ B. K. Meyer, H. Alves, D. M. Hofmann, W. Kriegseis, D. Forster, F. Bertram, J. Christen, A. Hoffmann, M. Straßburg, M. Dworzak, U. Haboeck, and A. V. Rodina, Phys. Status Solidi B 241, 231 (2004).

${ }^{17}$ D. C. Reynolds, C. W. Litton, and T. C. Collins, Phys. Rev. 140, A1726 (1965).

${ }^{18}$ F. Bertram, J. Christen, A. Dadgar, and A. Krost, Appl. Phys. Lett. 90, 041917 (2007).

${ }^{19}$ B. K. Meyer, J. Sann, S. Lautenschläger, M. R. Wagner, and A. Hoffmann, Phys. Rev. B 76, 184120 (2007).

${ }^{20}$ For a good review of these mechanisms, see M. Schirra, R. Schneider, A. Reiser, G. M. Prinz, M. Feneberg, J. Biskupek, U. Kaiser, C. E. Krill, K. Thonke, and R. Sauer, Phys. Rev. B 77, 125215 (2008).

${ }^{21}$ F. Tuomisto, V. Ranki, K. Saarinen, and D. C. Look, Phys. Rev. Lett. 91, 205502 (2003).

${ }^{22}$ A. Janotti and C. G. Van de Walle, Phys. Rev. B 76, 165202 (2007).

${ }^{23}$ P. J. Dean, Prog. Solid State Chem. 8, 1 (1973).

${ }^{24}$ K. Shahzad, B. A. Khan, D. J. Olego, and D. A. Cammack, Phys. Rev. B 42, 11240 (1990).

${ }^{25}$ G. F. Neumark, Phys. Rev. B 29, 1050 (1984).

${ }^{26}$ D. C. Look, J. Appl. Phys. 104, 063718 (2008). 